\title{
Olanzapine Attenuates the Okadaic Acid-Induced Spatial Memory Impairment and Hippocampal Cell Death in Rats
}

\author{
Jue He ${ }^{1,3}$, Yi Yang ${ }^{2,3}$, Haiyun Xu' ${ }^{1,3}$, Xia Zhang' and Xin-Min Li*,I \\ 'Neuropsychiatry Research Unit, Department of Psychiatry, University of Saskatchewan, Saskatoon, SK, Canada; ${ }^{2}$ Department of Neurology, \\ First Teaching Hospital, Jilin University, Chang Chun, China
}

\begin{abstract}
It is hypothesized that olanzapine, an atypical antipsychotic drug, has beneficial effects on cognitive impairment and neuropathological changes in treating neurodegenerative diseases. In the present study, we investigated the effects of chronic administration of olanzapine on the spatial memory impairment and hippocampal cell death induced by the direct injection of okadaic acid (OA), a potent neurotoxin, into the rat hippocampus. After being pretreated with olanzapine $(0.5$ or $2 \mathrm{mg} / \mathrm{kg} / \mathrm{day}$, i.p.) for 2 weeks, the rats were unilaterally microinjected with $\mathrm{OA}(100 \mathrm{ng})$ into the hippocampus, and then were continuously administrated with olanzapine for an additional week. The rats were trained on a spatial memory task in an eight-arm radial maze before OA administration, and tested on the same task $18 \mathrm{~h}$ after the last olanzapine injection. After the behavioral test, the rats were killed for Niss staining and terminal deoxynucleutidyl transferase-mediated biotinylated UTP nick end labeling staining. OA significantly induced spatial memory impairment, and caused pyramidal cell loss in the CAI and apoptotic cell death in the hippocampus. Olanzapine significantly attenuated OA-induced spatial memory impairment and the OA-induced neuropathological changes in the hippocampus. These findings suggest that olanzapine may have therapeutic effects in treatment of cognitive impairment and neuropathological changes of schizophrenia and other neurodegenerative diseases.

Neuropsychopharmacology (2005) 30, I 5 I I - I520, advance online publication, I I May 2005; doi: I 0.I 038/sj.npp. I 300757
\end{abstract}

Keywords: olanzapine; okadaic acid; memory; hippocampus; cell death; neuroprotection

\section{INTRODUCTION}

Neuroanatomical and clinical studies of schizophrenia suggest that progressive neuropathological changes (such as neuronal atrophy and/or cell death) occur over the course of the disease (Woods et al, 1990; Waddington et al, 1991; Arnold and Trojanowski, 1996; DeLisi et al, 1997). Cognitive deficits tend to occur early in the course of schizophrenia, and the severity of deficits is predictive of the long-term treatment outcome for patients (Green, 1996). Neural injury or neurodegeneration may cause cognitive deficits in schizophrenia (Kraepelin, 1919; Harvey, 1998).

Olanzapine, an atypical antipsychotic drug, has been widely used in the treatment of schizophrenia and other psychoses without causing agranulocytosis (Fulton and Goa, 1997). It effectively treats positive and negative

\footnotetext{
*Correspondence: Dr X-M Li, Neuropsychiatry Research Unit, Department of Psychiatry, University of Saskatchewan, 103 Wiggins Road, Saskatoon, SK, Canada SK S7N 5E4, Tel: + I 306966 8573, Fax: + I 306966 8830, E-mail: xin-min.li@usask.ca

3 These authors contributed equally to this work

Received 5 January 2005; revised 23 March 2005; accepted 24 March 2005

Online publication: I April 2005 at http://www.acnp.org/citations/ Npp040 I0505000 I/default.pdf
}

symptoms and has a low association with tardive dyskinesia or parkinsonism (Beasley et al, 1996, 1997; Casey, 1997). Olanzapine is also associated with wideranging benefits on cognitive functioning, and may effectively alleviate cognitive impairment in schizophrenia patients (Purdon et al, 2000; Cuesta et al, 2001; Bilder et al, 2002; Harvey et al, 2003). Furthermore, we have previously demonstrated that olanzapine is effective in reducing $\mathrm{PC} 12$ cell death induced by hydrogen peroxide, $\beta$-amyloid peptide, or $N$-methyl-4-phenylpyridinium ions $\left(\mathrm{MPP}^{+}\right)$ (Qing et al, 2003; Wei et al, 2003a,b). We have also shown that olanzapine effectively reduces the methamphetamine (METH)-induced mortality, and attenuates the METHinduced decrease of tyrosine hydroxylase immunoreactivity in the striatum in rats ( $\mathrm{He}$ et al, 2004). We hypothesized that olanzapine has beneficial effects on cognitive impairment and neuropathological changes in treating neurodegenerative diseases.

Okadaic acid (OA), a selective and potent inhibitor of the serine/threonine phosphatases 1 and $2 \mathrm{~A}$ (Ishihara et al, 1989; Cohen et al, 1990), has been reported to cause neuronal cell death in vitro (Cagnolli et al, 1996) and in vivo (Van Dam et al, 1998; He et al, 2001). OA may have rapid metabolic consequences leading to cell death by altering rates of phosphorylation-dephosphorylation in vivo (Arias 
et al, 1998). In cultured rat cortical neurons, OA may induce cell death by increasing phosphorylation of microtubuleassociated protein 2 and tau concomitant with early changes in the neuronal cytoskeleton (Arias et al, 1993). OA may also induce cellular injury and death by inactivating Bcl-2 by phosphorylation (Haldar et al, 1995), activating caspase3 (Hong et al, 2002), or forming high reactive free radicals (Matias et al, 1999) in vitro. Infusion of OA into the rat brain results in severe memory impairment, accompanied by remarkable neuropathological changes including hippocampal neurodegeneration, a paired helical filament-like phosphorylation of tau protein, and the formation of $\beta / \mathrm{A} 4$ amyloid containing plaque-like structures in gray and white matter areas (Arendt et al, 1995, 1998; Zhao et al, 1995; He et al, 2001; Tian et al, 2004).

In the present study, unilateral microinjection of OA into the dorsal hippocampus in rats was used as a memory impairment and hippocampal neurodegeneration animal model. The possible beneficial effect of olanzapine on the $\mathrm{OA}$-induced spatial working and reference memory impairment was examined in a radial arm maze task, and the neuroprotective potential of olanzapine on OA-induced lesion in the hippocampus was evaluated by Nissl staining and terminal deoxynucleutidyl transferase-mediated biotinylated UTP nick end labeling (TUNEL) staining.

\section{MATERIALS AND METHODS}

\section{Animals}

Male Sprague-Dawley rats (Charles River, St Constant, QC) weighing 225-250 g at the beginning of the experiments were used. They were housed four or five per cage with free access to food and water under controlled laboratory conditions (a 12:12 light: dark cycle at room temperature $\left.\left(19-21^{\circ} \mathrm{C}\right)\right)$. The rats were acclimated to the laboratory for 7 days before the experiments. All procedures with animals were performed in accordance with the guidelines established by the Canadian Council on Animal Care and were approved by the University of Saskatchewan Animal Care Committee.

\section{Preliminary Experiment}

In order to decide the dose of OA for olanzapine experiment, different doses $(0,50$, and $100 \mathrm{ng} / \mu \mathrm{l}$ saline $)$ of $\mathrm{OA}$ were injected into the right hippocampus (the same injection method described in Surgery and microinjection) after spatial reference/working memory task training in the rats (see Radial arm maze task). The rats were tested for spatial memory (see Radial arm maze task) 1 week after OA injection.

\section{Drug Treatment}

Olanzapine (Eli Lilly and Company, Indianapolis, IN) was freshly dissolved in $0.2 \%$. glacial acetic acid in saline. The ammonium salt of $\mathrm{OA}$ (Research Biochemicals International, Natick, MA) was dissolved to a concentration of $122 \mu \mathrm{M}(100 \mathrm{ng} / \mu \mathrm{l})$ in saline. The total experimental period was 22 days. The rats were pretreated with olanzapine $(0.5$ or $2 \mathrm{mg} / \mathrm{kg} / \mathrm{day}$ ) or $0.2 \%$ glacial acetic acid in saline as vehicle 1 by intraperitoneal (i.p.) injection once a day for 2 weeks. On day 15 , the rats were microinjected with $\mathrm{OA}$ (100 ng in $1 \mu \mathrm{l}$ saline) or saline (vehicle $2 ; 1 \mu \mathrm{l}$ ) into the right hippocamus (see Surgery and microinjection). At $1 \mathrm{~h}$ after the microinjection of $\mathrm{OA}$ or saline, the rats were continuously administrated with olanzapine $(0.5$ or $2 \mathrm{mg} /$ $\mathrm{kg} /$ day) or vehicle 1 by i.p. injection $(1.0 \mathrm{ml} / \mathrm{kg})$ once a day for 1 week. These procedures produced the following four groups of rats: vehicle $1+$ vehicle $2(\mathrm{CON})$, vehicle $1+\mathrm{OA}$ (OA), olanzapine $0.5 \mathrm{mg} / \mathrm{kg} / \mathrm{day}+\mathrm{OA}$ (OLA0.5 + OA), and olanzapine $2.0 \mathrm{mg} / \mathrm{kg} /$ day + OA (OLA2.0 + OA)

\section{Surgery and Microinjection}

On day 15, the rats were anesthetized with isoflurane inhalation $(2.0 \%$ isoflurane in oxygen for induction, $1.5 \%$ isoflurane in oxygen for maintenance), and restrained in a stereotactic apparatus. OA (100 ng in $1 \mu \mathrm{l}$ saline) or saline $(1 \mu \mathrm{l})$ was injected into the right dorsal hippocampus (A: $-3.8 \mathrm{~mm}$, L: $2.5 \mathrm{~mm}$ from bregma, and $\mathrm{V}: 3.2 \mathrm{~mm}$ ) according to the atlas of Paxinos and Watson (1986). OA or saline was injected over a 2 min period, and the injection cannula was left in place for an additional 5 min to allow for diffusion of OA away from the injection site. The dosage of OA (100 ng) was based on our preliminary data (Figure 1) and the published reports (Arias et al, 1998; He et al, 2001). The position of the injection site was examined in Nisslstained hippocampal sections. The rats that had injection sites away from the dorsal hippocampus were omitted from data analysis

\section{Radial Arm Maze Task}

The radial arm maze used in the present study consisted of eight arms, numbered from 1 to $8(48 \times 12 \mathrm{~cm})$, extending radially from a central area $(32 \mathrm{~cm}$ in diameter), with a $5 \mathrm{~cm}$ edge around the apparatus. The maze was placed $40 \mathrm{~cm}$ above the floor and was surrounded by various extramaze cues such as different laboratory benches and posters. Rats were trained for the spatial reference/working memory task, as described previously (Zou et al, 1998; Mizuno et al, 2000; He et al, 2002a, b). From day 2 to 5 , the rats were shaped in the maze to run to the end of the arms and consume the bait, in groups of four rats. The bait was initially available throughout the maze, but was gradually restricted to the food cup. Following this shaping period, each rat was placed individually in the center of the maze and subjected to the reference and working memory task training from day 6 to 14 (9 days, 3 trials/day with a 5 min interval). Finally, each rat was individually tested for the reference and working memory task on day 22 (1 week after OA or saline microinjection). The first training trial of each day and the test trial (on day 22) were performed $18 \mathrm{~h}$ after the injection of olanzapine or vehicle 1. Olanzapine administrated $18 \mathrm{~h}$ before the radial maze training and test did not evoke any sedating effect during maze experiments in rats. In all training and test trials, the same four arms (nos. 1, 2, 4, and 7) were baited. The other four arms (nos. 3, 5, 6, and 8 ) were never baited. The training or test trial continued until all four baits in the food cups had been consumed or 
until $5 \mathrm{~min}$ had elapsed. Reference memory is regarded as a long-term memory for information that remains constant over repeated trials (memory for the positions of baited arms), while working memory is considered as a short-term memory in which the information to be remembered changes in every trial (memory for the positions of arms that had already been visited in each trial; Olton et al, 1979). Thus, measures were made of the number of reference memory errors (entering an arm that was not baited) and working memory errors (entering an arm containing food but previously entered).

\section{Locomotor Speed and Food Consumption}

We measured locomotor speed and food consumption to see whether motor function and/or motivation were affected by the treatments ( $\mathrm{OA}$ and olanzapine), because the animals' motor function and motivation may have an impact on their performance in the radial arm maze task. Locomotor speed was calculated by total time/number of total entries in the test trial of the spatial memory task on day 22. On the same day (day 22), after the spatial memory test, rats were measured for food consumption. The rats were individually placed in a home cage, and then 10 baits, which were the same as the one used in the radial arm maze task, were provided. The time taken to consume all 10 baits in the food cup was recorded, with a cutoff time of $180 \mathrm{~s}$ (Zou et al, 1998).

\section{Nissl Staining and TUNEL Staining}

On day 22 , the rats were deeply anesthetized with chloral hydrate $(400 \mathrm{mg} / \mathrm{kg}$, i.p.) after the reference/working memory test, and were perfused through the ascending aorta with $250-300 \mathrm{ml}$ of $0.1 \mathrm{M}$ phosphate-buffered saline (PBS, pH 7.4) followed by $300-350 \mathrm{ml}$ of a fixative solution of $4 \%$ paraformaldehyde in PBS. The brains were removed, postfixed in the same fixative for $24 \mathrm{~h}$, and then cryoprotected in $30 \%$ sucrose in PBS. The brains were cut into $40-\mu \mathrm{m}$-thick coronal sections on a cryostat. The sections throughout the hippocampus were mounted on gelatincoated slides and allowed to air-dry overnight.

The surviving pyramidal cells in the hippocampus of rats were visualized by Nissl staining (He et al, 2001; Zhang et al, 2002). The mounted sections were rehydrated in distilled water, and submerged in $0.5 \%$ cresyl violet solution for about $1 \mathrm{~min}$ until the desired depth of staining was achieved.

Apoptotic nuclei showing nucleosomal DNA fragmentation can be detected by TUNEL staining (Gavrieli et al, 1992). The apoptotic cells in the hippocampus of rats were stained by a TUNEL staining kit according to the manufacturer's instructions (Trevigen, Gaithersburg, MD; Zhang et al, 2002). TUNEL staining relies on the detection of fragmented DNA strands, but because fragmentation can occur via nonapoptotic mechanisms, it is not absolutely specific for apoptosis (Wang et al, 2000).

\section{Cell Counting and Data Analysis}

The number of the CA1 pyramidal cells in Nissl staining sections (three sections of the right hemisphere of each rat at levels of $-3.60,-3.80$, and $-4.00 \mathrm{~mm}$; Paxinos and Watson, 1986) was counted at $\times 400$ magnification. Cells were counted only if structures of appropriate size and shape were demonstrated clearly. Questionable structures were examined under $\times 1000$ magnification and were not counted if identification remained uncertain. Similarly, the number of TUNEL-positive cells in the sections of the whole right hippocampus of each rat at levels of $-3.64,-3.84$, and $-4.04 \mathrm{~mm}$ (Paxinos and Watson, 1986) was also counted at $\times 400$ magnification. Although we did not use a stereological method for cell counting, our cell counting analysis provided complementary data to our qualitative observation on the hippocampal sections.

\section{Statistical Analysis}

All results were expressed as means \pm SEM. A two-tailed $t$-test for independent samples was used for the CON and OA group comparison. The significant difference was determined by a one-way ANOVA, followed by Bonferroni's test for multiple comparisons. The significant difference of the spatial memory formation before OA injection in the CON, OA, OLA0.5+OA, and OLA2.0+OA groups was determined by a two-way ANOVA factoring time (daily training of 9 days) and drug treatment (olanzapine). A $P$-value of less than 0.05 was regarded as statistically significant.

\section{RESULTS}

\section{Preliminary Experiment: OA (100 ng) Induced Spatial Memory Impairment in the Radial Arm Maze Task}

The number of working and reference memory errors measured premicroinjection and 1 week after microinjection in the saline-injected (CON), OA (50 ng)-injected (OA50), and OA (100 ng)-injected (OA100) groups is shown in Figure 1. One-way ANOVA showed that there was no difference in the number of working $(\mathrm{F}(2,12)=0.169$, $P=0.846)$ or reference $(\mathrm{F}(2,12)=0.300, P=0.746)$ memory errors on the last training day (premicroinjection) among the CON, OA50, and OA100 groups. However, one-way ANOVA showed that OA had an effect on the number of working $(\mathrm{F}(2,12)=7.176, P=0.009)$ and reference $(F(2,12)=17.765, P<0.001)$ memory errors after microinjection of OA, and a post hoc analysis with Bonferroni's test indicated that the number of working memory errors in the OA100 group was significantly higher than that in the CON group $(P<0.01$; Figure $1 \mathrm{a})$, and that the number of reference memory errors in the OA100 group was significantly higher than that in the CON group $(P<0.001 ;$ Figure $1 \mathrm{~b})$ and the OA50 group $(P<0.01$; Figure 1b).

\section{Chronic Administration of Olanzapine did not Affect the Spatial Memory Formation of Rats before OA Injection}

Figures $2 \mathrm{a}$ and $\mathrm{b}$ show the time course (total 9 days; from day 6 to 14) of working memory (Figure 2a) and reference memory (Figure 2b) formation during the training period of the radial arm maze task performed 
before OA injection. Two-way ANOVA disclosed that daily training (time) significantly decreased the number of both working $(\mathrm{F}(8,24)=39.707, P<0.001)$ and refer-
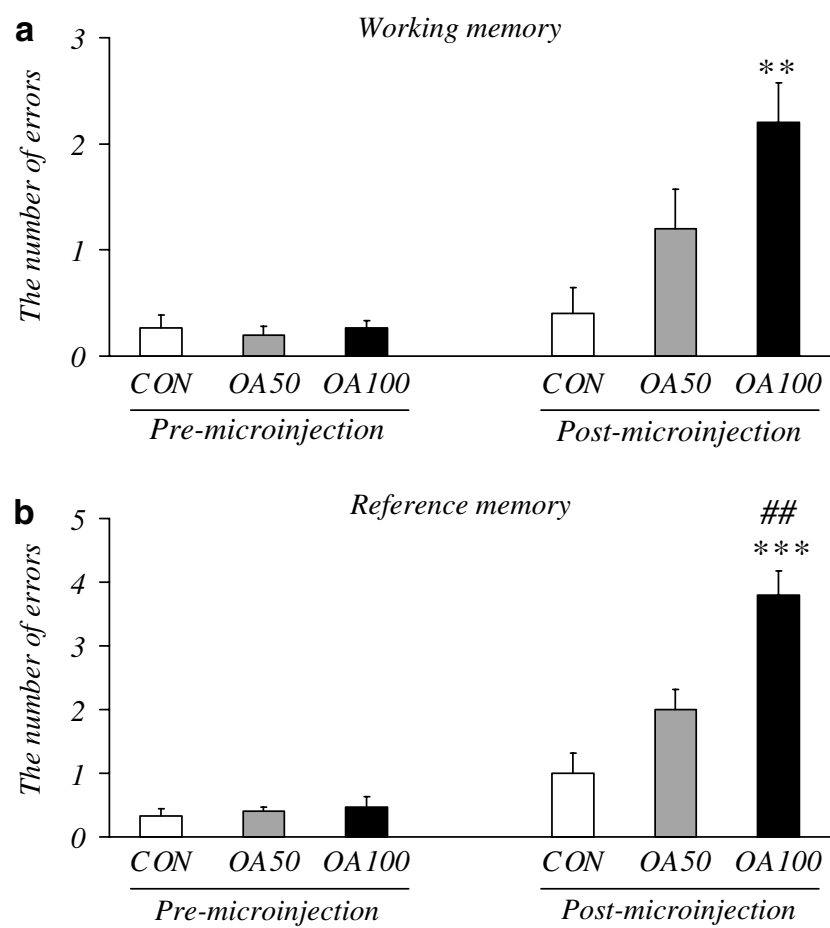

Figure I Dose effect of $O A$ on the working (a) and reference (b) memory in the radial arm maze task. The working and reference memory was measured I week after the microinjection of OA or saline into the right hippocampus. Results are expressed as means \pm SEM $(n=5$ in each group). $* * P<0.01$ and $* * * P<0.001$ vs $C O N$, and $\# \# P<0.01$ vs $O A 50$. ence $(F(8,24)=132.979, \quad P<0.001)$ memory errors, and that olanzapine produced no effect on the number of working $(\mathrm{F}(3,24)=1.606, \quad P=0.217) \quad$ or $\quad$ reference $(\mathrm{F}(3,24)=1.936, \quad P=0.153) \quad$ memory errors with no interaction between the two factors (time and olanzapine) in the number of working $(\mathrm{F}(8,24)=1.189$, $P=0.258)$ or reference $(\mathrm{F}(8,24)=0.966, P=0.513)$ memory errors. One-way ANOVA showed that there was no difference in the number of working $(\mathrm{F}(3,22)=0.612$, $P=0.614)$ or reference $(\mathrm{F}(3,22)=0.334, P=0.801)$ memory errors on the last training day among the CON, OA, OLA0.5 + OA, and OLA2.0 + OA groups before saline or $\mathrm{OA}$ injection.

\section{Chronic Administration of Olanzapine Attenuated the OA-Induced Spatial Memory Impairment in the Radial Arm Maze Task}

The number of working and reference memory errors in the rats was measured 1 week after $\mathrm{OA}$ or saline microinjection and the data are shown in Figures $2 c$ and d. A two-tailed $t$-test for independent samples disclosed that the number of working $(P<0.001$; Figure $2 c)$ and reference $(P<0.001$; Figure $2 \mathrm{~d})$ memory errors in the $\mathrm{OA}$ group is significantly higher than that in the CON. One-way ANOVA showed that the treatment (olanzapine) had an effect on the number of working $(\mathrm{F}(2,16)=5.094, \quad P=0.019)$ and reference $(\mathrm{F}(2,16)=12.827, P<0.001)$ memory errors in OA-injected rats, and a post hoc analysis with Bonferroni's test indicated that the number of working memory errors in the OLA2.0+ OA group is significantly lower than that in the OA group $(P<0.05$; Figure $2 \mathrm{c})$, and that the number of reference memory errors in the OLA2.0 + OA group is significantly a

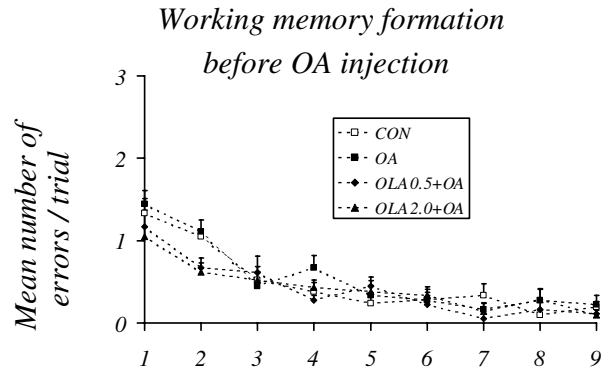

C

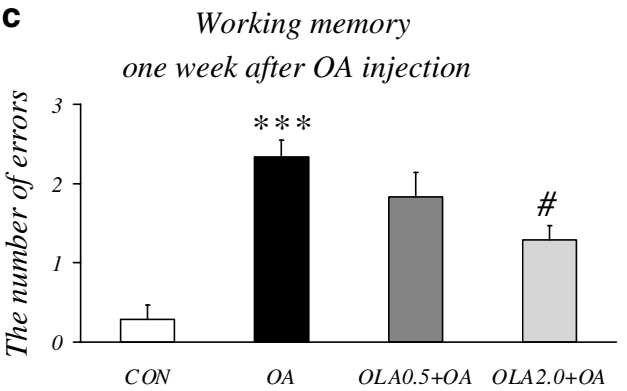

b Reference memory formation

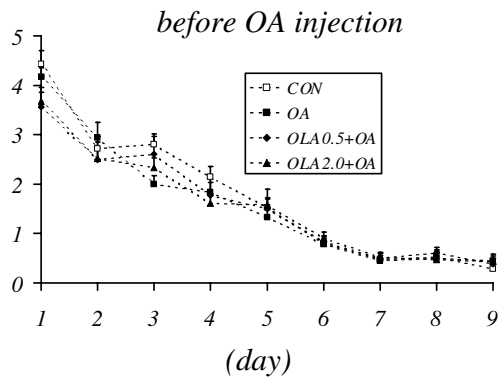

d

Reference memory one week after $O A$ injection

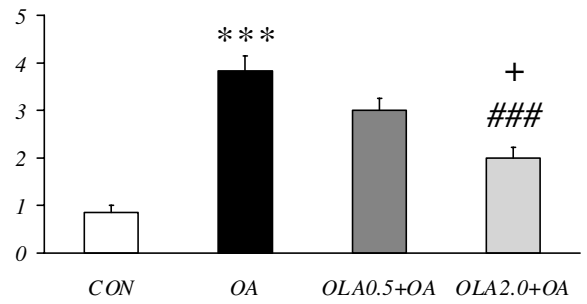

Figure 2 Olanzapine did not affect the spatial working (a) and reference (b) memory formation before OA or saline microinjection in the radial arm maze task in rats. However, olanzapine significantly attenuated the OA-induced impairment in animals' working (c) and reference (d) memory, which was measured by the radial arm maze task I week after the microinjection of OA or saline into the right hippocampus. Results are expressed as means \pm SEM ( $n=6-7$ in each group). ${ }^{*} * * P<0.001$ vs $C O N, \# P<0.05$ and $\# \# \# P<0.001$ vs $O A$, and $+P<0.05$ vs OLA0.5 $+O A$. 
lower than that in the OA group $(P<0.001$; Figure $2 \mathrm{~d})$ and the OLA0.5 + OA group $(P<0.05$; Figure $2 \mathrm{~d})$.

\section{Chronic Administration of Olanzapine and OA Injection had no Effect on Food Consumption and Locomotor Speed of the Rats}

The treatments (OA and olanzapine) had no effect on locomotor speed and food consumption in the rats. A twotailed $t$-test for independent samples disclosed that there was no difference in locomotor speed and food consumption between the OA and CON groups. One-way ANOVA showed that the treatment (olanzapine) had no effect on locomotor speed $(\mathrm{F}(2,16)=1.862, P=0.188)$ and food consumption $(\mathrm{F}(2,16)=0.316, P=0.734)$ in $\mathrm{OA}$-injected rats.

\section{Chronic Administration of Olanzapine Attenuated the OA-Induced Decrease in the Number of Surviving Pyramidal Cells in the CA1 Region of Hippocampus}

Nissl staining of hippocampal sections was used to evaluate the effect of olanzapine on the OA-induced decrease in the number of surviving pyramidal cells in the CA1 region of the injected hippocampus. Representative photomicrographs of Nissl staining in the hippocampus in each group are shown in Figure 3a.

As shown in Figure $3 \mathrm{~b}$, the number of surviving pyramidal cells in the CA1 region of the injected hippocampus was counted in each group. A two-tailed $t$-test for independent samples disclosed that the number of pyramidal cells in the CA1 region of the injected hippocampus significantly decreased in the OA group compared with that in the CON group $(P<0.001)$. One-

a
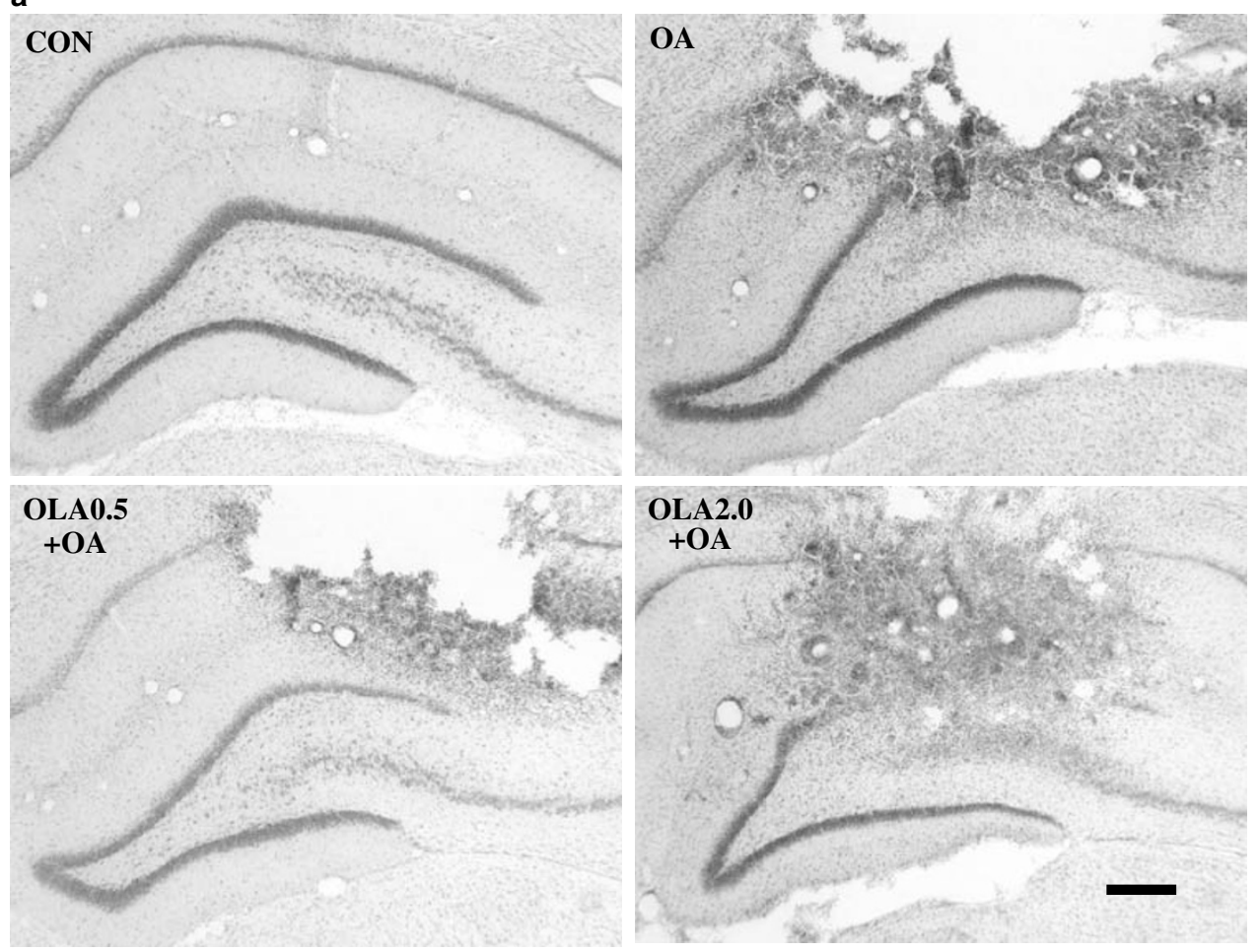

b

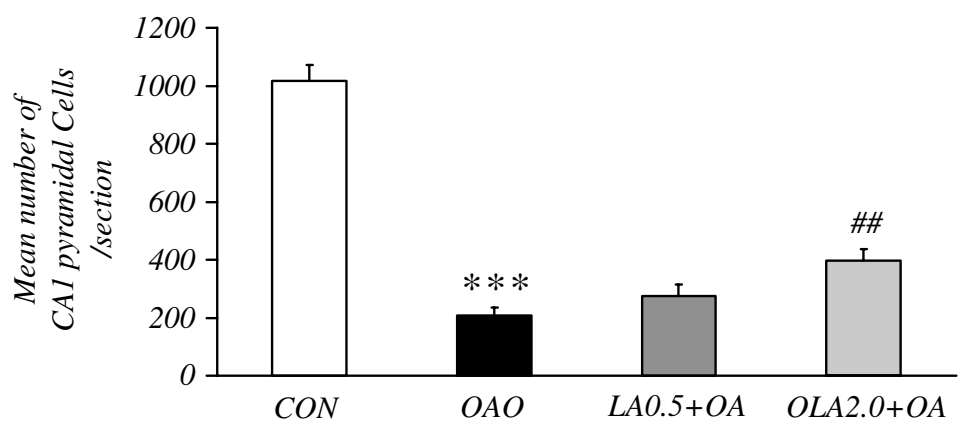

Figure 3 (a) Representative photomicrographs of Nissl staining in the injected hippocampus of the rats in the CON, OA, OLA0.5 + OA, and OLA2.0 + OA groups. In the OA-injected hippocampi (OA, OLA0.5 + OA, and OLA2.0 + OA), the lesion is obvious. The lesion in the rats treated with OA alone $(O A)$ is more extensive, fragile, and easier to be peeled off when the brains were cut compared with that in the rats treated with both olanzapine and $O A$ (OLA2.0 + OA). The scale bar is $300 \mu \mathrm{m}$. (b) Quantitative analysis of the effect of olanzapine on the OA-induced pyramidal cell loss in the CAI region of hippocampus. The number of CAI pyramidal cells in Nissl staining sections was counted at $\times 400$ magnification. Results are expressed as means \pm SEM ( $n=6-7$ in each group). $* * * P<0.001$ vs $C O N$ and $\# \# P<0.01$ vs $O A$. 
way ANOVA showed that the treatment (olanzapine) had an effect $(\mathrm{F}(2,16)=7.489, P=0.005)$ in the OA-injected rats, and a post hoc analysis with Bonferroni's test indicated that the number of pyramidal cells in the CA1 region of the injected hippocampus in the OLA2.0 $+\mathrm{OA}$ group is significantly higher than that in the OA group $(P<0.01)$.

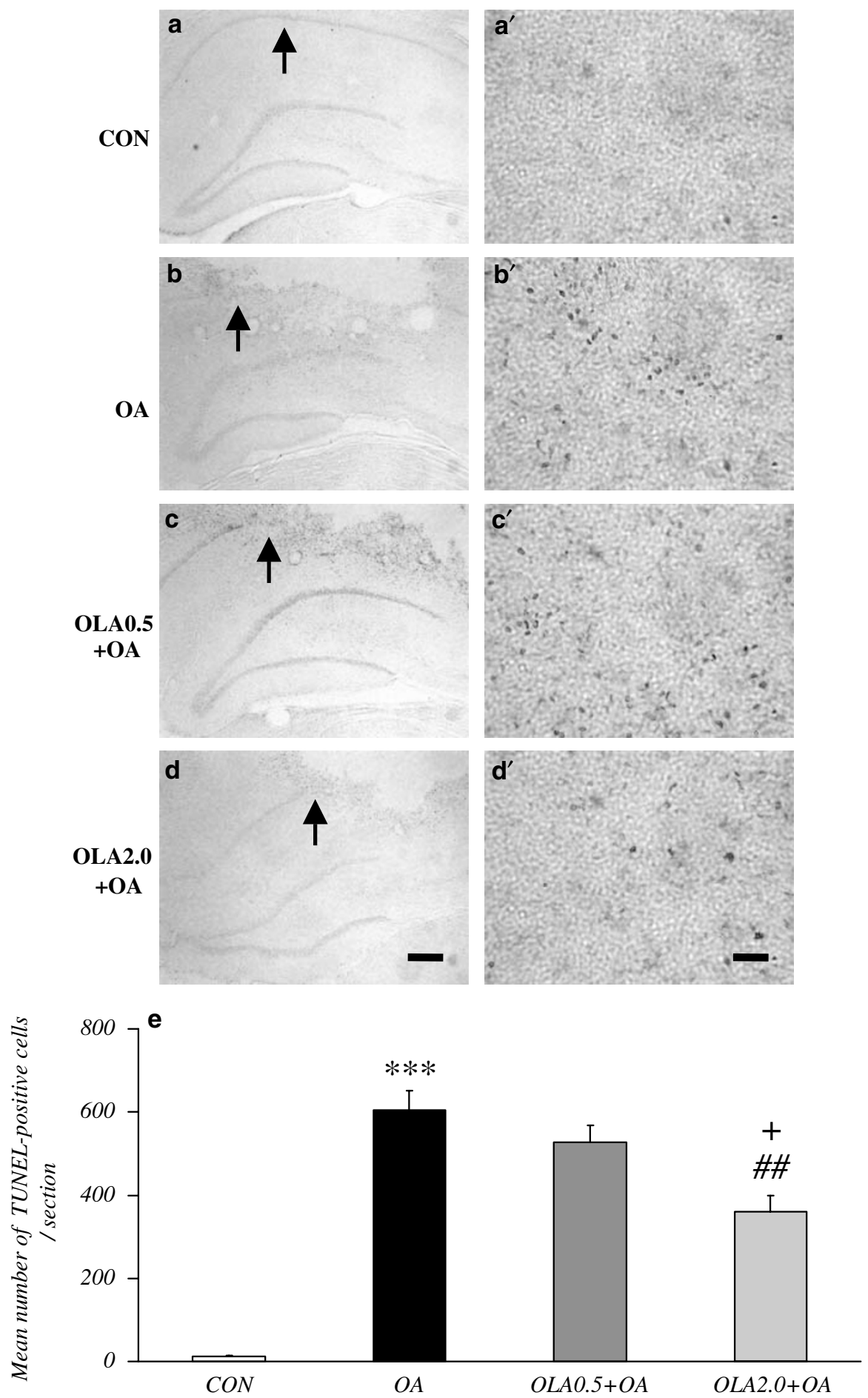

Figure $4\left(a-d, a^{\prime}-d^{\prime}\right)$ Representative photomicrographs of TUNEL staining in the injected hippocampus of the rats in the CON (a, $\left.a^{\prime}\right), O A(b, b),, O L A 0.5$ $+\mathrm{OA}\left(\mathrm{c}, \mathrm{c}^{\prime}\right)$, and OLA2.0 + OA $\left(\mathrm{d}, \mathrm{d}^{\prime}\right)$ groups. The high magnification of right photomicrographs $\left(\mathrm{a}^{\prime}, \mathrm{b}^{\prime}, \mathrm{c}^{\prime}\right.$, and $\left.\mathrm{d}^{\prime}\right)$ were picked up from $a, b, c$, and $d_{1}$ respectively. The arrows on the low-magnification panels indicate the location of the high-magnification images. In the hippocampi of OA-injected groups (b, $\left.b^{\prime} ; c, c^{\prime} ; d, d^{\prime}\right)$, the TUNEL-positive cells are visible in different frequency, whereas there are almost no TUNEL-positive cells in the hippocampus of the control rat $\left(a, a^{\prime}\right)$. The scale bar represents $300 \mu \mathrm{m}$ in a-d, and represents $30 \mu \mathrm{m}$ in $a^{\prime}-d^{\prime}$. (e) Quantitative analysis of the effect of olanzapine on the OAinduced increase of TUNEL-positive cells in the injected hippocampus. The number of TUNEL-positive cells in the hippocampus was counted at $\times 400$ magnification. Results are expressed as means $\pm \operatorname{SEM}(n=6-7$ in each group). $* * * P<0.00$ I vs CON, \#\#P<0.0 Ivs OA, and $+P<0.05$ vs OLA0.5 + OA. 


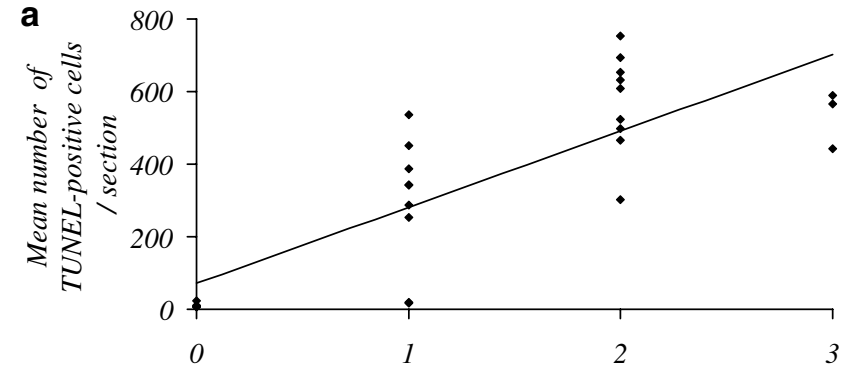

The number of working memory errors

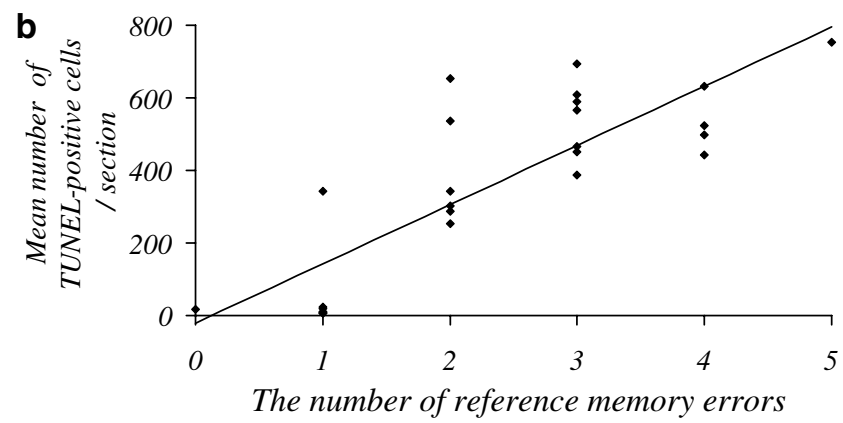

Figure 5 Scatter plot of the number of working (a) or reference (b) memory errors against the number of TUNEL-positive cells in the hippocampus after $O A$ or saline injection in each individual rat in the $C O N$, OA, OLA0.5 + OA, and OLA2.0 + OA groups ( $n=6-7$ in each group).

\section{Chronic Administration of Olanzapine Attenuated the Number of OA-Induced TUNEL-Positive Cells in the Hippocampus}

The hippocampal sections prepared by TUNEL staining were used to evaluate the effect of olanzapine on OAinduced apoptotic cell death in the injected hippocampus. Representative photomicrographs of TUNEL staining in the hippocampus in each group are shown in Figures $4 a-d$. Under a higher magnification $(\times 400)$, many TUNELpositive cells were observed in the hippocampus of the OA-injected rats (Figures $4 \mathrm{~b}^{\prime}, 4 \mathrm{c}^{\prime}$, and $4 \mathrm{~d}^{\prime}$ ) but not in that of the CON rats (Figure $4 \mathrm{a}^{\prime}$ ).

As shown in Figure 4e, the number of TUNEL-positive cells in the injected hippocampus was counted in each group. A two-tailed $t$-test for independent samples disclosed that the number of TUNEL-positive cells in the injected hippocampus significantly increased in the OA group compared with that in the CON group $(P<0.001)$. Oneway ANOVA showed that the treatment (olanzapine) had an effect $(\mathrm{F}(2,16)=9.247, P=0.002)$ in the OA-injected rats, and a post hoc analysis with Bonferroni's test indicated that the number of TUNEL-positive cells in the injected hippocampus in the OLA2.0+OA group is significantly lower than that in the OA group $(P<0.01)$ and that in the OLA0.5 + OA group $(P<0.05)$.

\section{Spatial Memory Errors Increased with the Increasing Number of TUNEL-Positive Cells in the Hippocampus}

The relationship between the number of working memory errors and the number of TUNEL-positive cells in the hippocampus was significant $\left(r^{2}=0.6292, \quad P<0.0001\right.$;
Figure 5a). There was also a significant direct relationship between the number of reference memory errors and the number of TUNEL-positive cells in the hippocampus $\left(r^{2}=0.6835, P<0.0001\right.$; Figure $\left.5 b\right)$.

\section{DISCUSSION}

Memory impairment induced by microinjection of $\mathrm{OA}$ into animal brains has been reported (Arendt et al, 1995; He et al, 2001; Bennett et al, 2003). Similarly, in the present study, unilateral microinjection of OA (100 ng) into the dorsal hippocampus induced impairment in spatial working and reference memory. Consistent with the previous reports (Arias et al, 1998; He et al, 2001; Ramirez-Munguia et al, 2003), Nissl staining in the present study showed that the direct microinjection of $\mathrm{OA}$ into the dorsal hippocampus robustly decreased the number of surviving pyramidal neurons in the CA1 region of the hippocampus. Microinjection of $\mathrm{OA}$ into the hippocampus also induced apoptosis as revealed by TUNEL staining. This is in accordance with previous studies that showed the OAinduced apoptosis in HL60 leukemia (Riordan et al, 1998), neuroblastoma (Cabado et al, 2004), and mammalian cells (Boe et al, 1991). Because the intact hippocampus is required for the recall, item recognition, and associative recognition memory of animals (Alvarado and Bachevalier, 2005; Sweatt, 2004), the OA-induced spatial memory impairment may be attributed, at least in part, to the hippocampal cell death caused by the drug.

Chronic administration of olanzapine significantly attenuated the OA-induced spatial memory impairment. This improvement is parallel to the alleviative effect of olanzapine on the OA-induced cell death in the hippocampus, suggesting an association between the neuroprotective effect and the memory-improving effect exerted by olanzapine.

The attenuating effect of chronic administration of olanzapine on the OA-induced neurodegeneration and apoptosis provides the direct evidence supporting the neuroprotective action of olanzapine. Although the mechanisms responsible for the neuroprotective effect remain to be elucidated, our previous studies have provided some insights into this issue. In PC12 cells, olanzapine is effective in reducing the cell death induced by $\mathrm{H}_{2} \mathrm{O}_{2}, \mathrm{~A} \beta$, or $\mathrm{MPP}^{+}$, which may cause oxidative stress thus killing the treated cells (Qing et al, 2003; Wei et al, 2003a, b). We demonstrated that these cytoprotective actions in the cells might involve the upregulation of superoxide dismutase-1, one of the endogenous antioxidant enzymes, by the drug ( $\mathrm{Li}$ et al, 1999; Qing et al, 2003; Wei et al, 2003a). In addition, olanzapine can regulate the translocation and expression of pro- and antiapoptotic proteins $\mathrm{Bax}$ and $\mathrm{Bcl}-\mathrm{X}_{\mathrm{L}}$ in $\mathrm{PC} 12$ cells (Wei et al, 2003b). In animal studies, chronic administration of olanzapine was shown to upregulate the expression of Bcl-2 and BDNF in the hippocampus (Bai et al, 2003, 2004) and to help restore the repeated restrain stress-induced decrease in these two neuroprotective proteins in hippocampal neurons (Luo et al, 2004). Furthermore, the prevention of $\mathrm{Bcl}-2$ decrease has been associated with the protective effects of olanzapine on methamphetamine-induced neurotoxicity (He et al, 2004). 
Thus our own previous data, together with the findings by other investigators that OA-induced apoptosis is associated with downregulation of $\mathrm{Bcl}-2$ and can be prevented by upregulation of Bcl-2 (Benito et al, 1997; Nuydens et al, 2000; Cabado et al, 2004), suggest that Bcl-2 plays an important role in the neuroprotective effects of olanzapine on OA-induced neurodegeneration and apoptosis. Olanzapine may also attenuate OA-induced neurotoxicity by upregulating superoxide dismutase (Manna et al, 1998; Li et al, 1999; Matias et al, 1999), and perform protective effects on OA-induced apoptotic cell death by modulating the expression of pro- and antiapoptotic proteins such as Bax and Bcl-X $\mathrm{L}_{\mathrm{L}}$ (Wei et al, 2003b; Cabado et al, 2004). However, further studies are necessary to elucidate whether olanzapine attenuated OA-induced neurotoxicity by directly affecting the activation of phosphatases or caspases.

The time point ( 1 week after OA injection) for spatial memory measurement ruled out the direct effect of OA (as an inhibitor of PP1 and PP2A) on memory processes in the present study (He et al, 2001; Bennett et al, 2003). It is likely that OA-induced spatial memory impairment in the present paradigm is due to the secondary effect of OA-induced hippocampal cell death (He et al, 2001). Unilateral microinjection of OA $(100 \mathrm{ng})$, which induced spatial memory impairment, induced a massive lesion of the normal brain tissue (Figure $3 \mathrm{a}$ ) in the OA-injected rats. The lesion in the rats treated with OA alone was more extensive, fragile, and easier to be peeled off when the brains were cut on a cryostat compared with that in the rats treated with both olanzapine and OA (Figure 3a). Chronic olanzapine not only significantly attenuated OA-induced decrease of the number of pyramidal cells in the CA1 region, but also attenuated OA-induced increase in apoptotic cell death in the hippocampus. Therefore, the improving effects of olanzapine on OA-induced spatial memory impairment in rats may be subsequent to its attenuating effects on OAinduced hippocampal cell death.

It should be noted that there must be some molecules linking the attenuating effects of OA-induced hippocampal cell death (neuroprotective effect) and memory improvement. Among possible molecules, BDNF is a stronger candidate. It has been shown that chronic administration of olanzapine upregulates the expression of BDNF mRNA in the hippocampus (Bai et al, 2003), and improves memory in different maze tasks in animal studies (Nowakowska et al, 1999; Wolff and Leander, 2003). Indeed, the involvement of BDNF in spatial memory formation and maintenance in a radial arm maze test has been demonstrated in rats (Mizuno et al, 2000; Radecki et al, 2005).

Chronic administration of olanzapine and $\mathrm{OA}$ injection had no effect on locomotor speed and food consumption of the animals. This result rules out the possibility that olanzapine may affect motor function or/and motivation for food thus improving their performance in the radial arm maze task. However, it is still possible that OA-induced behavioral deficits are not limited to memory deficits, and the capacity of olanzapine to reverse other types of behavioral deficits remains unknown.

Although the above explanation is helpful for us to understand the mechanisms responsible for the improving effect of olanzapine on the OA-induced memory impairment, there are other experimental data meriting our attention. It was reported that injection of $\mathrm{OA}$ into the nucleus basalis of Meynert, which is a major source of cholinergic innervation of the cerebral cortex and hippocampus, induced a decreased level of acetylcholine (Ach) in the brain and spatial memory deficit in rats (Tian et al, 2004). On the other hand, olanzapine in rats induces an increase of Ach release in the medial prefrontal cortex and hippocampus, a possible factor contributing to cognitive improvement in schizophrenia (Ichikawa et al, 2002; Shirazi-Southall et al, 2002). Therefore, the effects of olanzapine on Ach may be another possible contributor to its improving effect on the OA-induced memory impairment.

Although chronic administration of olanzapine (14 days, 0.5 or $2 \mathrm{mg} / \mathrm{kg} /$ day) had no effects on spatial memory formation (Figures $2 \mathrm{a}$ and $\mathrm{b}$ ), we still cannot completely exclude the possibility that olanzapine itself affects memory performance because there was no olanzapine control group (chronic olanzapine treatment (21 days, 0.5 or $2 \mathrm{mg} / \mathrm{kg} /$ day) + intrahippocampal vehicle microinjection) in the present study. However, because of the length of the training and the interval between the training and test, it is possible that even if olanzapine by itself had some memory-enhancing effect, this effect could not be detected in the olanzapine control group. In this context, the alternative possibility that olanzapine may reverse OA effect on memory through a nonspecific memory-enhancing effect should be considered. Moreover, further studies are necessary to elucidate whether the effects of olanzapine observed in the present model system are also shared by other atypical antipsychotic drugs.

In conclusion, unilateral microinjection of OA significantly induced spatial working and reference memory impairment, and caused neurodegeneration and apoptosis in the injected hippocampus. Chronic pre- and postlesion administration of olanzapine effectively attenuated OAinduced spatial memory impairment and the OA-induced neuropathological changes in the hippocampus. These findings suggest that olanzapine may have therapeutic effects in treatment of cognitive impairment and neuropathological changes of schizophrenia and other neurodegenerative diseases.

\section{ACKNOWLEDGEMENTS}

This work was supported by the Saskatchewan Health Research Foundation Postdoctoral Fellowship to Jue He. We thank Dr Augusto V Juorio and Dr Sergey Fedoroff for their helpful comments during the preparation of this manuscript. We are also grateful to Gabriel Stegeman for her excellent technical assistance.

\section{REFERENCES}

Alvarado MC, Bachevalier J (2005). Selective neurotoxic damage to the hippocampal formation impairs performance of the transverse patterning and location memory tasks in rhesus macaques. Hippocampus 15: 118-131.

Arendt T, Holzer M, Fruth R, Bruckner MK, Gartner U (1995). Paired helical filament-like phosphorylation of tau, deposition of B/A4-amyloid and memory impairment in rat induced by 
chronic inhibition of phosphatase 1 and 2A. Neuroscience 69: 691-698.

Arendt T, Holzer M, Fruth R, Bruckner MK, Gartner U (1998). Phosphorylation of tau, Aß-formation, and apoptosis after in vivo inhibition of PP-1 and PP-2A. Neurobiol Aging 19: 3-13.

Arias C, Becerra-Garcia F, Arrieta I, Tapia R (1998). The protein phosphatase inhibitor okadaic acid induces heat shock protein expression and neurodegeneration in rat hippocampus in vivo. Exp Neurol 153: 242-254.

Arias C, Sharma N, Davies P, Shafit-Zagardo B (1993). Okadaic acid induced early changes in microtubule-associated protein 2 and tau phosphorylation prior to neurodegeneration in cultured cortical neurons. J Neurochem 61: 673-682.

Arnold SE, Trojanowski JQ (1996). Recent advances in defining the neuropathology of schizophrenia. Acta Neuropathol 92: 217-231.

Bai O, Chlan-Fourney J, Bowen R, Keegan D, Li XM (2003). Expression of brain-derived neurotrophic factor mRNA in rat hippocampus after treatment with antipsychotic drugs. J Neurosci Res 71: 127-131.

Bai O, Zhang H, Li XM (2004). Antipsychotic drugs clozapine and olanzapine upregulate bcl-2 mRNA and protein in rat frontal cortex and hippocampus. Brain Res 1010: 81-86.

Beasley CM, Sanger T, Satterlee W, Tollefson GD, Tran PV, Hamilton S (1996). Olanzapine $v s$. placebo: results of a double-blind, fixeddose olanzapine trial. Psychopharmacology 124: 159-167.

Beasley CM, Tollefson GD, Tran PV (1997). Efficacy of olanzapine: an overview of pivotal clinical trials. J Clin Psychiatry 58: 7-12.

Benito A, Lerga A, Silva M, Leon J, Fernandez-Luna JL (1997). Apoptosis of human myeloid leukemia cells induced by an inhibitor of protein phosphatases (okadaic acid) is prevented by Bcl-2 and Bcl-X(L). Leukemia 11: 940-944.

Bennett PC, Moutsoulas P, Lawen A, Perini E, Ng KT (2003). Novel effects on memory observed following unilateral intracranial administration of okadaic acid, cyclosporin A, FK506 and [ $\left.\mathrm{MeVal}^{4}\right]$ CyA. Brain Res 988: 56-58.

Bilder RM, Goldman RS, Volavka J, Czobor P, Hoptman M, Sheitman B et al (2002). Neurocognitive effects of clozapine, olanzapine, risperidone, and haloperidol in patients with chronic schizophrenia or schizoaffective disorder. Am J Psychiatry 159: 1018-1028.

Boe R, Gjertsen BT, Vintermyr OK, Houge G, Lanotte M, Doskeland SO (1991). The protein phosphatase inhibitor okadaic acid induces morphological changes typical of apoptosis in mammalian cell. Exp Cell Res 195: 237-246.

Cabado AG, Leira F, Vieytes MR, Vieites JM, Botana LM (2004). Cytoskeletal disruption is the key factor that triggers apoptosis in okadaic acid-treated neuroblastoma cells. Arch Toxicol 78: 74-85.

Cagnolli CM, Kharlamov E, Atabay C, Uz T, Manev H (1996). Apoptosis induced in neuronal cultures by either the phosphatase inhibitor okadaic acid or the kinase inhibitor staurosporine is attenuated by isoquinolinesulfonamides $\mathrm{H}-7, \mathrm{H}-8$ and $\mathrm{H}-9$. J Mol Neurosci 7: 65-76.

Casey DE (1997). The relationship of pharmacology to side effects. J Clin Psychiatry 58: s55-s62.

Cohen P, Holmes CF, Tsukitani Y (1990). Okadaic acid: a new probe for the study of cellular regulation. Trends Biol Sci 15: 98-102.

Cuesta MJ, Peralta V, Zarzuela A (2001). Effects of olanzapine and other antipsychotics on cognitive function in chronic schizophrenia: a longitudinal study. Schizophr Res 48: 17-28.

DeLisi LE, Sakuma M, Rew W, Kushner M, Hoff AL, Grimson R (1997). Schizophrenia as a chronic active brain process: a study of progressive brain structural change subsequent to the onset of schizophrenia. Psychiatry Res 74: 129-140.

Fulton B, Goa KL (1997). Olanzapine. A review of its pharmacological properties and therapeutic efficacy in the management of schizophrenia and related psychoses. Drugs 53: 281-298.
Gavrieli Y, Sherman Y, Ben-Sasson SA (1992). Identification of programmed cell death in situ via specific labeling of nuclear DNA fragmentation. J cell Biol 119: 493-501.

Green MF (1996). What are the functional consequences of neurocognitive deficits in schizophrenia? Am J Psychiatry 153: 321-330.

Haldar S, Jena N, Croce CM (1995). Inactivation of Bcl-2 by phosphorylation. Proc Natl Acad Sci USA 92: 4507-4511.

Harvey PD (1998). Schizophrenia and related disorders in late life. In: Bellack AS, Hersen M (eds). Comprehensive Clinical Psychology. Elsevier: Amsterdam. Vol 8, pp 74-86.

Harvey PD, Green MF, McGurk SR, Meltzer HY (2003). Changes in cognitive functioning with risperidone and olanzapine treatment: a large-scale, double-blind, randomized study. Psychopharmacology 169: 404-411.

He J, Xu H, Yang Y, Zhang X, Li XM (2004). Neuroprotective effects of olanzapine on methamphetamine-induced neurotoxicity are associated with an inhibition of hyperthermia and prevention of Bcl-2 decrease in rats. Brain Res 1018: 186-192.

He J, Yamada K, Nabeshima T (2002a). A role of Fos expression in the CA3 region of the hippocampus in spatial memory formation in rats. Neuropsychopharmacology 26: 259-268.

He J, Yamada K, Zou L, Nabeshima T (2001). Spatial memory deficit and neurodegeneration induced by the direct injection of okadaic acid into the hippocampus in rats. J Neural Transm 108: 1435-1443.

He J, Yamada K, Zou L, Nakajima A, Kamei H, Nabeshima T (2002b). Learning and memory in two different reward tasks in a radial arm maze in rats. Behav Brain Res 134: 139-148.

Hong HN, Yoon SY, Suh J, Lee JH, Kim D (2002). Differential activation of caspase-3 at two maturational stages during okadaic acid-induced rat neuronal death. Neurosci lett 334: 63-67.

Ichikawa J, Dai J, O’Laughlin IA, Fowler WL, Meltzer HY (2002). Atypical, but not typical, antipsychotic drugs increase cortical acetylcholine release without an effect in the nucleus accumbens or striatum. Neuropsychopharmacology 26: 325-339.

Ishihara H, Martin BL, Brautigan DL, Karaki H, Ozaki H, Kato Y et al (1989). Calyculin A and okadaic acid: inhibitors of protein phosphatase activity. Biochem Biophys Res Commun 159: 871-877.

Kraepelin E (1919). Dementia Praecox and Paraphrenia. E \& S Livingstone: Edinburgh.

Li X-M, Chlan-Fourney J, Bennett V, Shrikhande S, Juorio AV, Keegan D et al (1999). Differential effects of Olanzapine on the gene expression of superoxide dismutase and low affinity nerve growth factor receptor. J Neurosci Res 56: 72-75.

Luo C, Xu H, Li XM (2004). Post-stress changes in BDNF and Bcl-2 immunoreactivities in hippocampal neurons: effect of chronic administration of olanzapine. Brain Res 1025: 194-202.

Manna SK, Zhang HJ, Yan T, oberley LW, Aggarwal BB (1998). Overexpression of manganese superoxide dismutase suppresses tumor necrosis factor-induced apoptosis and activation of nuclear transcription factor-kappaB and activated protein-1. J Biol Chem 273: 13245-13254.

Matias WG, Traore A, Bonini M, Sanni A, Creppy EE (1999). Oxygen reactive radicals production in cell culture by okadaic acid and their implication in protein synthesis inhibition. Hum Exp Toxicol 18: 634-639.

Mizuno M, Yamada K, Olariu A, Nawa H, Nabeshima T (2000). Involvement of BDNF in spatial memory formation and maintenance in a radial arm maze test in rats. J Neurosci 20: 7116-7121.

Nowakowska E, Chodera A, Kus K (1999). Influence of olanzapine on cognitive functions and catalepsy in rats after single and chronic administration. Pol J Pharmacol 51: 295-300.

Nuydens R, Dispersyn G, Van Den Keiboom G, de Jong M, Connors R, Ramaekers $\mathrm{F}$ et al (2000). Bcl-2 protects against 
apoptosis-related microtubule alterations in neuronal cells. Apoptosis 5: 43-51.

Olton DS, Becker JT, Handelmann GE (1979). Hippocampus, space, and memory. Behav Brain Sci 2: 313-365.

Paxinos G, Watson C (1986). The Rat Brain in Stereotaxic Coordinates. Academic Press: London.

Purdon SE, Jones BDW, Stip E, Labelle A, Addington D, David SR et al (2000). Neuropsychological change in early phase schizophrenia during 12 months of treatment with olanzapine, risperidone, or haloperidol. Arch Gen Psychiatry 57: 249-258.

Qing H, Xu H, Wei Z, Gibson K, Li XM (2003). The ability of atypical antipsychotic drugs $v s$ haloperidol to protect PC12 cells against $\mathrm{MPP}^{+}$-induced apoptosis. Eur J Neurosci 17: 1563-1570.

Radecki DT, Brown LM, Martinez J, Teyler TJ (2005). BDNF protects against stress-induced impairments in spatial learning and memory and LTP. Hippocampus 15: 246-253.

Ramirez-Munguia N, Vera G, Tapia R (2003). Epilepsy, neurodegeneration, and extracellular glutamate in the hippocampus of awake and anesthetized rats treated with okadaic acid. Neurochem Res 28: 1517-1524.

Riordan FA, Foroni L, Hoffbrand AV, Mehta AB, Wickremasinghe RG. (1998). Okadaic acid-induced apoptosis of HL60 leukemia cells is preceded by destabilization of bcl-2 mRNA and downregulation of bcl-2 protein. FEBS Lett 435: 195-198.

Shirazi-Southall S, Rodriguez DE, Nomikos GG (2002). Effects of typical and atypical antipsychotics and receptor selective compounds on acetycholine efflux in the hippocampus of the rat. Neuropsychopharmacology 26: 583-594.

Sweatt JD (2004). Hippocampal function in cognition. Psychopharmacology 174: 99-110.

Tian Q, Lin Z-Q, Wang X-C, Chen J, Wang Q, Gong C-X et al (2004). Injection of okadaic acid into the meynert nucleus basalis of rat brain induces decreased acetylcholine level and spatial memory deficit. Neuroscience 126: 277-284.
Van Dam A-M, Bol JGJM, Binnekade R, Van Muiswinkel FL (1998). Acute or chronic administration of okadaic acid to rats induces brain damage rather than Alzheimer-like neuropathology. Neuroscience 85: 1333-1335.

Waddington JL, O'Callaghan E, Buckley P, Larkin C, Redmond O, Stack J et al (1991). The age differences of MRI abnormalities in schizophrenia suggest early ventricular enlargement but later prominence of cortical atrophy. Schizophr Res 5: 188-189.

Wang C, Kaufmann JA, Sanchez-Ross MG, Johnson KM (2000). Mechanisms of $N$-methyl-D-aspartate-induced apoptosis in phencyclidine-treated cultured forebrain neurons. J Pharmacol Exp Ther 294: 287-295.

Wei Z, Bai O, Richardson JS, Mousseau DD, Li X-M (2003a). Olanzapine protects PC12 cells from oxidative stress induced by hydrogen peroxide. J Neurosci Res 73: 364-368.

Wei Z, Mousseau DD, Richardson JS, Dyck LE, Li X-M (2003b). Atypical antipsychotics attenuate neurotoxicity of $\beta$-amyloid (25-35) by modulating $\mathrm{Bax}$ and $\mathrm{Bcl}-\mathrm{X}_{\mathrm{L} / \mathrm{S}}$ expression and localization. J Neurosci Res 74: 942-947.

Wolff MC, Leander JD (2003). Comparison of the effects of antipsychotics on a delayed radial maze task in the rat. Psychopharmacology 168: 410-416.

Woods BT, Yurgelun-Todd D, Benes FM, Frankenburg FR, Pope HG, McSparren J (1990). Progressive ventricular enlargement in schizophrenia: comparison to bipolar disorder and correlation with clinical course. Biol Psychiatry 27: 341-352.

Zhang X, Cui SS, Wallace AE, Hannesson DK, Schmued LC, Saucier DM et al (2002). Relations between brain pathology and temporal lobe epilepsy. J Neurosci 22: 6052-6061.

Zhao WQ, Bennett P, Sedman GL (1995). The impairment of longterm memory formation by the phosphatase inhibitor okadaic acid. Brain Res Bull 36: 557-561.

Zou L-B, Yamada K, Tanaka K, Kameyama T, Nabeshima T (1998). Nitric oxide synthase inhibitors impair reference memory formation in a radial arm maze task in rats. Neuropharmacology 37: 323-330. 